\title{
A Prospective Comparison of Vertebral Column Decancellation Versus Pedicle Subtraction Osteotomy in Thoracolumbar Kyphosis
}

By Yunfei Ouyang, Wang Yan, Zheng Guoquan, Xiao Songhua, Huang Peng \& Zhang Xuesong

Abstract- Study Design: A prospective study.

Summary of Background Data: For advanced stages of ankylosing spondylitis (AS), the correction of spine deformities is quite often with pedicle subtraction osteotomy (PSO). We reported a new resected technique of spinal osteotomy, called the vertebral column decancellation (VCD) in 2010 to treat rigid scoliosis and severe sharp angular spinal deformities. We first report comparisons between VCD with PSO.

Objective: We performed to compare VCD and PSO in correcting kyphosis deformities related to AS.

Keywords: ankylosing spondylitis; kyphosis; vertebral column decancellation (VCD); Osteotomy; posterior approach.

GJMR-H Classification: NLMC Code: WE 725

Strictly as per the compliance and regulations of:

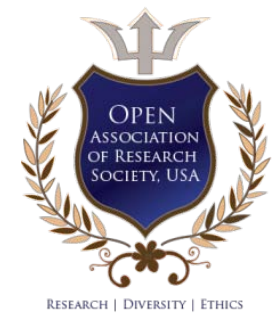

(c) 2020. Yunfei Ouyang, Wang Yan, Zheng Guoquan, Xiao Songhua, Huang Peng \& Zhang Xuesong. This is a research/review paper, distributed under the terms of the Creative Commons Attribution-Noncommercial 3.0 Unported License http://creativecommons.org/licenses/by-nc/3.0/), permitting all non-commercial use, distribution, and reproduction in any medium, provided the original work is properly cited. 


\title{
A Prospective Comparison of Vertebral Column Decancellation Versus Pedicle Subtraction Osteotomy in Thoracolumbar Kyphosis
}

\author{
Yunfei Ouyang ${ }^{\alpha}$, Wang Yan ${ }^{\sigma}$, Zheng Guoquan ${ }^{\circ}$, Xiao Songhua ${ }^{\omega}$, Huang Peng ${ }^{*} \&$ Zhang Xuesong ${ }^{\S}$
}

\begin{abstract}
Study Design: A prospective study.
Summary of Background Data. For advanced stages of ankylosing spondylitis (AS), the correction of spine deformities is quite often with pedicle subtraction osteotomy (PSO). We reported a new resected technique of spinal osteotomy, called the vertebral column decancellation (VCD) in 2010 to treat rigid scoliosis and severe sharp angular spinal deformities. We first report comparisons between VCD with PSO.
\end{abstract}

Objective: We performed to compare VCD and PSO in correcting kyphosis deformities related to AS.

Methods: Seven patients underwent VCD, and nine underwent PSO (4 male, 12 female; mean age 37.9 years; range, 23-65 years). We performed preoperative and postoperative imaging examinations and recorded intraoperative and postoperative general complications. We analyzed radiographic results, complications, and patient satisfaction over a mean follow-up of 3.35 years (range, 9 0.9-3.5.1 years).

Results: The angle correction obtained by a one-segment VCD was significantly, and larger than PSO $(40+4$ vs. $25+5$ degrees; $p<0001)$. Similarly, the height of the resected vertebral posterior wall after correction by VCD was larger than after PSO $(13.3+2.6$ vs. $7.7+2.8 \mathrm{~mm} ; \mathrm{p}=0.01)$. Operative time and blood loss were slightly less after VCD. The global kyphosis correction was not different between the two groups $(P=0.9333)$, nor was the mean Oswestry disability index (ODI) at the final follow-up.

Conc/usions: Single-stage, posterior VCD is a proper option to manage severe kyphosis secondary to AS. A single-segment VCD obtains a larger correction than PSO. VCD maintained more of the height of the resected vertebra, which shortened the middle column more than after PSO. A better radiographic correction was noted in the VCD and PSO groups.

Keywords: ankylosing spondylitis; kyphosis; vertebral column decancellation (VCD); Osteotomy; posterior approach.

\section{Key Points}

1. The vertebral column decancellation (VCD) and the pedicle subtraction osteotomy (PSO) are two kinds of techniques for correcting the spinal kyphotic deformity.

Corresponding Author a: M.D., Department of orthopedics, no. 920 hospital, Kunming City, Yunnan province, CHINA.

e-mail: yunfeioyang@hotmail.com

Corresponding Author o: Ph.D., Orthopedic ward 3, 16th floor, surgery building, 301 hospital, no. 28 fuxing road, Beijing, CHINA.

e-mail:yanwang301@163.com

Author $\rho \omega ¥ \xi$ : Orthopedic ward 3, 16th floor, surgery building, 301 hospital, no. 28 fuxing road, Beijing, CHINA.
2. We found VCD has some advantages of decreasing the vertebra column shorten, shortening operation time, lower blood loss, and lower expends. A singlesegment VCD obtains a larger correction than PSO.

3. VCD adds one more choice for surgeons correcting severe spinal kyphosis safely.

Mini Abstract- The pedicle subtraction osteotomy (PSO) is commonly used in spinal surgery. We first reported the vertebral column decancellation (VCD) in 2010. There have been no reports comparing VCD and PSO; here we show our experience, the techniques, and outcomes at 3.35 years comparing VCD and PSO, which in correcting kyphosis deformities.

\section{INTRODUCTION}

$\Lambda$ nkylosing spondylitis (AS) is an inflammatory disorder that can cause a variety of debilitating lorthopedic problems. Among the many musculoskeletal manifestations of AS, spinal deformity is perhaps the most disabling to the patient. If the flexion deformity is excessive, the patient's field of vision is limited to the area near the feet, and walking is extremely difficult. Respiration becomes almost completely diaphragmatic.

Gastrointestinal symptoms resulting from pressure of the costal margin on the contents of the upper abdomen are common.3 In addition to improvement in function, the aesthetic improvement in appearance made by correcting the deformity is important to the patient. If extreme, the deformity should be corrected in two or more stages because of contracture of soft tissues and the danger of injury to the aorta, the inferior vena cava, and the major nerves to the lower extremities.

Pedicle subtraction osteotomy (PSO) as a close wedge osteotomy (CWO) is used most commonly to manipulate deformities related to AS. A single-stage, posterior osteotomy can often provide adequate correction while minimizing the pressure of injury to vascular and retroperitoneal structures positioned anterior to the spine. Traditionally, one can usually achieve 10 degrees of correction with each Smith Petersen osteotomy (SPO), and 30 degrees with a PSO4. Therefore, neither SPO nor PSO in one segment would be expected to achieve adequate correction for patients with severe kyphosis related to AS. 
Polysegmental osteotomies have been suggested for these deformities 5. Vertebral column decancellation (VCD) is a new spinal osteotomy technique for correction of sharp angular spinal deformities first reported in 2011 by our group.2 We have continued to further develop this VCD osteotomy technique in the lumbar spine to manage kyphosis deformities in AS. As a close-opening wedge osteotomy (COWO), theoretically VCD can result in a larger singlestage correction than PSO (Figure 1). Because there have been no reports comparing VCD and PSO, here we report our experience, techniques, and outcomes at 3.6 years comparing VCD and PSO in correcting kyphosis deformities related to AS.

\section{Materials And Methods}

From January 2009 to December 2013, 16 patients (13 males; 3 females; mean age, 37 years; range,23-51years) with kyphosis secondary to AS underwent operative treatment at ourinstitution. All patients signed the operation informed consent based on the understanding of the operation theoretically. The 16 patients were randomly divided into two groups by a third-party numerical table method without the knowledge of the surgeon or the patient. Seven patients underwent VCD, and nine underwent PSO. The operative technique of VCD includes resection of the posterior elements of 2 adjacent vertebrae, resection of the inferior-posterior aspect of the proximal vertebra, and the superior-posterior aspect of the distal vertebra, Followed by posterior instrumentation/stabilization with pedicle screws and spinal fusion. Preoperative and postoperative osteotomized vertebra height, lumbar lordosis Cobb angle, C7 plumb line, sagittal vertical axis (SVA), and sagittal Cobb angle of the osteotomized segment were documented. The operation time, blood loss, and general complications were documented.

\section{a) Surgical Technique}

For the PSO resection, we reported previously a method of transpedicular, bivertebral wedge osteotomy and discectomy to manage the sagittal plane deformity in patients with AS who have chin-brow vertical angles greater than $90^{\circ} .{ }^{6}$ Fiberoptic intubation preceded the induction of general anesthesia. The patients were placed in the prone position on the operating table, which was flexed in a reverse $V$ shape. Soft protective sponge mats were placed under the chest and abdomen. A midline skin incision was made, and the posterior elements of the lumbar spine were exposed by subperiosteal dissection as far laterally as the transverse processes. Pedicle screws were inserted into several segments above and below the osteotomy level under C-arm fluoroscopic guidance. Laminectomy and facetectomy at the osteotomy site were performed. Then, through the pedicle into the vertebral body and a high-speed drill was used to enlarge the hole. The transverse processes were excised at their bases. With a drill the cancellous bone of the inferior-posterior aspect of the upper vertebra and the superior-posterior aspect of lower vertebra were resected to create a cavity. The posterior and lateral cortex of the body was then resected with angled curettes. The vertebral canal was enlarged by resecting the residual proximal and distal lamina (dome decompression) to avoid compressing the dura while closing the osteotomy. We used to perform two-level PSO procedures for correcting kyphosis beyond $50^{\circ}$, because a one-level PSO can correct only about $30-40^{\circ}$ of kyphosis.

(Figure 6) Usually, $1 \mathrm{~mm}$ of resected posterior bone will equate to approximately $1^{\circ}$ of lordosis once the osteotomy is closed. During the closure of the osteotomy, the pedicle screws that were connected to the temporary short rod were loosened, and compressive forces were applied on the adjacent screws. After the osteotomy site was completely closed, a precontoured rod spanning the entire segments was fixed to the screws and tightened. On the other side, the temporary short rod was removed and replaced with a long permanent rod. Finally, bilateral rods were tightened to the pedicle screws, and the spine was stabilized in the corrected position. This was the standard PSO procedure.

For the VCD procedure, pedicle screws were inserted into 4 or 5 segments similar to the PSO procedure. A larger laminectomy was performed in 2-3 segments to obtain enough correction. Decancellation of the vertebral body was performed with a Y-shaped osteotomy. First, we created a smaller wedge at the significant direction of convex side vertebra using a high-speed drill, with the depth of the wedge being less than one second of the vertebral width. Then, we performed a line-section cut from the vertex of the wedge to the concave side. The approach for creating the small wedge can be transpedical or transvertebral (Figure 2). For the case in Figure 4, we used a transvertebral approach. The cortex of the concave side was weakened rather than resected, which conveniently prevented translation of the osteotomy section. The smaller wedge and the line-section compose the $\mathrm{Y}$ shaped osteotomy, which is a $360^{\circ}$, circumferential decancellation. The two surfaces of the wedge are closed and osteoclasis of the concave cortex occurred during correction of the kyphosis (Figure 2). The VCD is an incomplete resection of vertebral decancellation; a part of cancellous bone and the posterior vertebral posterior that were preserved serve the role of "Bony cage", which prevents excessive shortening of the spinal cord during the correction of the kyphosis. We consciously extend the distance between the upper, and lower pedicle screws of the osteotomized vertebra in case this "Bony cage" collapses during correction of the kyphosis. 


\section{b) Radio Radiographic assessment}

Full-length anteroposterior and lateral radiographs in the standing position obtained preoperatively and postoperatively, were used to make radiographic comparisons. In the sagittal plane, we measured the distance between the C7 plumb line and the posterior superior corner of $\mathrm{S} 1$, lumbar lordosis from L1 to S1, and global kyphosis. The height variation of the middle column was regarded as the height variation of the posterior wall of the resected vertebral body. We assessed the degree of middle column shortening by comparing the height of the posterior wall of the resected vertebra before and after surgery.

\section{c) Statistical analysis}

The SPSS software 18.0 was used for statistical analysis. Comparison between VCD and PSO was made using students t-test. The differences were regarded as significant when $\mathrm{P}<0.05$. Continuous data are expressed as mean + standard deviation.

\section{RESULTS}

\section{a) Surgical results}

One-level osteotomy was performed in all VCD cases $(n=7)$. For the PSO cases, the most frequent level of osteotomy was L3 followed by L1, and 5 cases underwent two-level osteotomies $(n=9)$. In the VCD group, the mean operation time was 297 min (range, 180- $540 \mathrm{~min}$ ), while for the PSO group, the mean operating room time was 325 min (range, 241-610 min). The average estimated blood loss was 2,400 ml (range, $1,200-5,000 \mathrm{ml}$ ) in VCD group and 2,800 $\mathrm{ml}$ (range, $1,860-6,000 \mathrm{ml}$ ) in PSO group. Compared with PSO, VCD resulted in a shorter operative time and less bleeding $(\mathrm{P}<0.05$ each).

\section{b) Radiographic results}

The basic demographic and radiographic data are list in Table 1. Significant differences were observed between the 2 groups in terms of the corrective contribution and the postoperative height of the posterior wall of the resected vertebra (Table 2). In the VCD group, the one level osteotomized mean angle was $40+4$ degrees while for the PSO group it was $25+5$. The corrective contribution of $1 \mathrm{VCD}$ is nearly equal to 1.5 PSO in correction of the kyphotic angle at the osteotomy site $(P<0.05)$. The height of the posterior wall of the resected vertebra after correction in the VCD group was greater than in the PSO group $(13.3+2.6$ vs $7.7+2.8 \mathrm{~mm} ; \mathrm{P}=0.0010$ ). The postoperative height of VCD was $\geq 1 / 2$ the preoperative height itself compared to $\leq 1 / 3$ in the PSO group (Figure 3 ). The preoperative and postoperative global kyphosis, the SVA and lumbar lordosis did not show significant differences between the 2 groups $(P>0.05)$. Bony healing area was observed by one year follow up radiographs and CTs in patient underwent VCD osteotomy (Figure 7).

\section{c) Complications}

Two patients in the VCD group and 3 patients in the PSO group experienced perioperative complications. In the VCD group, sagittal subluxation of the segments caudal to the osteotomy site was observed in 1 patient during the closure of the $\mathrm{L} 2$ osteotomy. Dural tear secondary to adhesions with the ossified ligamentum flavum occurred in 1 patient in PSO. One patient in the VCD group developed a paralytic ileus and one in the PSO group developed a transient neurologic deficit. No vascular complications were observed in either group.

\section{d) Outcome analysis}

At the final follow-up, the mean Oswestry disability index (ODI) was 30 (range 5-51) in the VCD group and 28 (range, 8-37) in the PSO group and was not significantly different between the two groups.

\section{Discussion}

The first SPO was described in 1945 by SmithPetersen as a one or two-level osteotomy for deformities of AS.8 A PSO is performed by removing the posterior elements and pedicles and decancellating the vertebral body, which then hinges on the anterior cortex.

For patients with severe, rigid, thoracolumbar kyphosis, a single PSO will accomplish approximately $30^{\circ}$ to $40^{\circ}$ correction, but still leaving a residual kyphosis of large magnitude postoperatively. 7,10 Therefore, it is often necessary to perform a PSO at 2 or 3 segments if the required correction exceeds $40^{\circ}$; Figure 6 shows a 23-year-old male with AS in whom we performed a twolevel (L1/L3) PSO. However, two PSOs result in substantial blood loss, extra surgical trauma, and the potential for more neurologic complications. 10 If the kyphosis is corrected by more than $40^{\circ}$ in these closing wedge osteotomy procedures, the spinal cord may become too long for the shortened vertebral column and may be curved, kinked, and potentially damaged because the hinge of the correction is positioned at the anterior longitudinal ligament at the apex of the deformity.

Moreover, a PSO is not universally successful in achieving the best sagittal correction. In contrast, COWO may be considered in patients with severe thoracolumbar kyphosis secondary to AS who require a large magnitude of correction with the anticipated correction being much more than $35^{\circ}$ after a 1-level osteotomy. ${ }^{11}$ VCD is one type of COWO, which is an incomplete resection of vertebral decancellation, and a part of the bone is preserved to serve as a "Bony cage" to prevent excessive spinal cord bucking during correction. The position of the bony cage is due to the $3-$ dimensional status of the deformities; therefore, VCD can be considered as a flexible osteotomy for multiplanar deformities both with coronal and sagittal imbalance, especially for rigid spinal deformities. 
Current techniques of vertebral column resection (VCR) may result in greater correction. During the close-opening procedure, the anterior column is opened and lengthened; simultaneously, the center of correction is moved posterior to achieve the greater sagittal correction, and the posterior and middle columns are shortened. If necessary, a strut autograft or a rectangular mesh packed with bone graft can be inserted through the posterior approach into the intervertebral gap to provide interbody support ${ }^{12,13,17,20}$ Kawahara and colleagues concluded that a bone correction exceeding $40^{\circ}$ or $50^{\circ}$ can be achieved by COWO without compromising the integrity of the spinal cord. ${ }^{12}$ Lenke et al reported 147 consecutive pediatric VCRs performed by 7 surgeons and demonstrated excellent radiographic correction. The posterior VCR was shown to be safe and effective in this large series of patients. These complex reconstructions, however, were associated with a 59\% complication rate, thus emphasizing the challenging nature of these patients and procedures. ${ }^{14}$

In the elderly patient, PSO and VCR can restore sagittal and coronal balance and substantial improvement in quality of life, but both techniques can lead to serious complications and should be used selectively. ${ }^{15}$ Complete and circumferential resection of one vertebra at a single level allows for tremendous correction quality in both the sagittal and coronal planes in a controlled fashion without the need for more than one more segment to be osteotomized. In AS, correction of sagittal plane deformities can be achieved by lengthening the anterior elements, shortening the posterior elements, or a combination of the two. Usually, coronal imbalance is accompanied by sagittal imbalance.

A failed operation for deformity or a congenital disorder can result in a rigid coronal imbalance.9 In the VCD procedure, however, the hinge shifts to the posterior aspect of the spinal cord in contrast to the PSO correction which is achieved by passive extension of the lumbar spine to close the posterior osteotomy and with an anterior hinge. Internal transpedicular fixation has been used to ensure immediate stability and rapid consolidation. ${ }^{16,17} \mathrm{VCD}$ correction is achieved with a more posterior hinge. A single level VCD for thoracolumbar kyphosis can result in up to $40^{\circ}-70^{\circ}$ of correction; the Y-shaped osteotomy of the VCD is one type of COWO that effectively decreases the shortening of the middle vertebra column. The position of the bony cage is due to the 3-dimensional status of the deformity; therefore, VCD is a flexible osteotomy for multiplanar deformities with both coronal and sagittal imbalance, and especially so for rigid spinal deformities (Figure 8 4). This advantage of a VCD allows a greater degree of correction than PSO. Although a VCR is regarded as allowing the greatest correction, ${ }^{18}$ VCR needs interbody implants to maintain the height of middle column ${ }^{13}$. VCD adds one more choice for surgeons correcting severe spinal kyphosis safely.

\section{AbBreviations}

AS - Ankylosing Spondylitis

COWO - Close-opening Wedge Osteotomy

CWO - Close Wedge Osteotomy

ODI - Oswestry Disability Index

PSO - Pedicle Subtraction Osteotomy

SPO - Smith-Petersen Osteotomy

VCD - Vertebral Column Decancellation

SVA - Sagittal Vertical Axis

SPSS - Statistical Product and Service Solutions

VCR - Vertebral Column Resection

\section{References Références Referencias}

1. Wang $Y$, Zhang $Y G$, Zheng GQ, Xiao SH, Zhang XS, Wang $Z$. Vertebral column decancellation for the management of rigid scoliosis: the effectiveness and safety analysis. Zhonghua Wai Ke Za Zhi. 2010; 48(22):1701-4.

2. Wang Y, Lenke LG. Vertebral column decancellation for the management of sharp angular spinal deformity. Eur Spine J. 2011; 20(10):1703-10.

3. Simmons EH. Kyphotic deformity of the spine in ankylosing spondylitis. Clin Orthop Relat Res. 1977(128):65-77.

4. Van Royen BJ, de Kleuver M, Slot GH. Polysegmental lumbar posterior wedge osteotomies for correction of kyphosis in ankylosing spondylitis. Eur Spine J. 1998; 7(2):104-10.

5. Thiranont N, Netrawichien P. Transpedicular decancellation closed wedge vertebral osteotomy for treatment of fixed flexion deformity of spine in ankylosing spondylitis. Spine (Phila Pa 1976). 1993; 18(16):2517-22.

6. Wang $Y$, Zhang $Y$, Mao K, Zhang X, Wang Z, Zheng $\mathrm{G}$, et al. Transpedicular bivertebrae wedge osteotomy and discectomy in lumbar spine for severe ankylosing spondylitis. J Spinal Disord Tech. 2010; 23(3):186-91.

7. Gill JB, Levin A, Burd T, Longley M. Corrective osteotomies in spine surgery. J Bone Joint Surg Am. 2008; 90(11):2509-20.

8. Smith-Petersen MN, Larson CB, OE A. Osteotomy of the spine for correction of flexion deformity in rheumatoid arthritis. J Bone Joint Surg Br. 1945; 27:1-11.

9. Lawrence G Lenke, Cho W. Vertebral Osteotomies-Review of Current Concepts. US Musculoskeletal Review. 2010; 5(1):46-9.

10. Kiaer T, Gehrchen M. Transpedicular closed wedge osteotomy in ankylosing spondylitis: results of surgical treatment and prospective outcome analysis. Eur Spine J. 2010; 19(1):57-64. 
11. Chang KW, Leng X, Zhao W, Ching-Wei C, Chen TC, Chang KI, et al. Quality control of reconstructed sagittal balance for sagittal imbalance. Spine (Phila Pa 1976). 2011; 36(3):E186-97.

12. Kawahara N, Tomita K, Baba H, Kobayashi T, Fujita $\mathrm{T}$, Murakami $\mathrm{H}$. Closing-opening wedge osteotomy to correct angular kyphotic deformity by a single posterior approach. Spine (Phila Pa 1976). 2001; 26(4): 391-402.

13. Rajasekaran S, Vijay K, Shetty AP. Single-stage closing-opening wedge osteotomy of spine to correct severe post-tubercular kyphotic deformities of the spine: a 3-year follow-up of 17 patients. Eur Spine J. 2010; 19(4):583-92.

14. Lenke LG, Newton PO, Sucato DJ, Shufflebarger $\mathrm{HL}$, Emans JB, Sponseller PD, et al. Complications after 147 consecutive vertebral column resections for severe pediatric spinal deformity: a multicenter analysis. Spine (Phila Pa 1976). 2013; 38(2):119-32.

15. Hassanzadeh $H$, Jain $A$, El Dafrawy $M H$, Ain $M C$, Mesfin A, Skolasky RL, et al. Three-Column Osteotomies in the Treatment of Spinal Deformity in Adult Patients 60 Years Old and Older: Outcome and Complications. Spine (Phila Pa 1976). 2012.

16. Thomasen E. Vertebral osteotomy for correction of kyphosis in ankylosing spondylitis. Clin Orthop Relat Res. 1985(194):142-52.

17. Scudese VA, Calabro JJ. Vertebral Wedge Osteotomy. Correction of Rheumatoid (Ankylosing) Spondylitis. JAMA. 1963; 186:627-31.

18. Cho WJ, Kang CN, Park YS, Kim HJ, Cho JL. Surgical correction of fixed kyphosis. Asian Spine J. 2007; 1(1):12-8.

Table 1: Patient demographics. * indicates that the patient underwent total hip replacement (THR)

\begin{tabular}{|c|c|c|c|c|c|c|c|c|c|c|c|}
\hline \multirow[t]{2}{*}{ Group } & \multirow[t]{2}{*}{ Cases } & \multirow[t]{2}{*}{ Sex } & \multirow[t]{2}{*}{ age } & \multirow{2}{*}{$\begin{array}{l}\text { Site of } \\
\text { Osteotomy }\end{array}$} & \multirow{2}{*}{$\begin{array}{l}\text { Osteotomy } \\
\text { angle } \\
\left({ }^{\circ}\right)\end{array}$} & \multicolumn{2}{|c|}{ SVA (cm) } & \multicolumn{2}{|c|}{$\begin{array}{l}\text { Lumbar lordosis } \\
\left({ }^{\circ}\right)\end{array}$} & \multicolumn{2}{|c|}{$\begin{array}{l}\text { Vertebra Height } \\
\text { (mm) }\end{array}$} \\
\hline & & & & & & Pre & Post & Pre & Post & Pre & Post \\
\hline \multirow{7}{*}{ VCD } & 1 & $\mathrm{~F}$ & 49 & L1 & 42 & 15 & 0 & 39 & -66 & 17 & 14 \\
\hline & 2 & $M$ & 43 & L3 & 32 & 19 & 5 & 36 & -50 & 21 & 12 \\
\hline & 3 & M & 48 & L3 & 38 & 28 & 9 & 36 & -53 & 22 & 11 \\
\hline & $4^{\star}$ & $M$ & 51 & L2 & 42 & 20 & 5 & 33 & -76 & 25 & 18 \\
\hline & 5 & M & 36 & L2 & 42 & 11 & 1 & 3 & -51 & 22 & 12 \\
\hline & 6 & $\mathrm{M}$ & 35 & L2 & 45 & 90 & 35 & 3 & -47 & 30 & 15 \\
\hline & 7 & M & 24 & L2 & 38 & 72 & 20 & 2 & -36 & 20 & 11 \\
\hline \multirow{9}{*}{ PSO } & 8 & $\mathrm{M}$ & 41 & L2 & 40 & 21 & 0 & 12 & -56 & 26 & 6 \\
\hline & $9^{*}$ & M & 35 & $\mathrm{~L} 1 / 3$ & $16 / 30$ & 28 & 8 & 30 & -33 & 22/22 & $18 / 8$ \\
\hline & 10 & F & 42 & $L 1 / 3$ & $28 / 21$ & 30 & 5 & 42 & -60 & 20/22 & $8 / 8$ \\
\hline & 11 & M & 34 & L2 & 24 & 80 & 50 & 0 & -42 & 22 & 8 \\
\hline & 12 & M & 24 & L3 & 32 & 100 & 80 & -2 & -48 & 23 & 10 \\
\hline & 13 & M & 25 & $L 1 / 3$ & $21 / 37$ & 25 & 3 & 28 & -30 & 22/22 & $6 / 3$ \\
\hline & 14 & M & 41 & L1 & 30 & 15 & 4 & 5 & -45 & 23 & 7 \\
\hline & 15 & $\mathrm{M}$ & 23 & T12/L2 & 20/22 & 150 & 60 & 3 & -27 & 23/25 & $6 / 13$ \\
\hline & 16 & F & 34 & L2 & 21 & 100 & 60 & 15 & -58 & 20 & 8 \\
\hline
\end{tabular}


Table 2: Radiographic data comparing PSO and VCD

\begin{tabular}{|c|c|c|c|c|c|c|c|c|c|}
\hline Group & $\begin{array}{l}\text { Pre/Post } \\
\text { operation }\end{array}$ & $\begin{array}{l}\text { Osteotmized } \\
\text { vertebral angle } \\
\text { (degrees) }\end{array}$ & $\begin{array}{c}\text { Height of } \\
\text { vertebral } \\
\text { posterior wall }\end{array}$ & $\begin{array}{l}\text { Lumbar } \\
\text { lordosis } \\
\text { (degrees) }\end{array}$ & $\begin{array}{l}\text { Global } \\
\text { kyphosis } \\
\text { (need }\end{array}$ & SVA (cm) & $\begin{array}{c}\text { GK mean } \\
\text { correction } \\
(\%)\end{array}$ & $\begin{array}{c}\text { Mean } \\
\text { correction for } \\
\text { every vertebra }\end{array}$ & $\begin{array}{c}\text { Mean umber of } \\
\text { resected } \\
\text { vertebra }\end{array}$ \\
\hline \multirow{2}{*}{$\begin{array}{c}\text { PSO } \\
(n=21) \\
\end{array}$} & Pre op & 0 & $22 \pm 4$ & $13( \pm 16)$. & $88( \pm 17)$ & $17.7( \pm 8.8)$ & \multirow[t]{2}{*}{$54 \%$} & \multirow[t]{2}{*}{32 vertebra } & \multirow[t]{2}{*}{$32 / 21(1.524)$} \\
\hline & Post op & $25( \pm 5)$ & $7.7( \pm 3)$ & $-42( \pm 12)$ & $41( \pm 10)$ & $5.3( \pm 1.6$ & & & \\
\hline \multirow[b]{2}{*}{$(n=20)$} & Pre op & 0 & $22( \pm 1)$ & $22( \pm 18)$ & $84( \pm 13.1)$ & $16.3( \pm 7.1)$ & \multirow[t]{2}{*}{$49 \%$} & \multirow[t]{2}{*}{ 39/vertebra } & \multirow[t]{2}{*}{$21 / 20(1.050)$} \\
\hline & Post op & $40( \pm 4)$ & $13( \pm 3)$ & $-54( \pm 13)$ & $43( \pm 12)$ & $3.2( \pm 3.1)$ & & & \\
\hline$P$ & & $p<0.01$ & 0.01 & 0.11 & 0.93 & 0.37 & & & \\
\hline
\end{tabular}
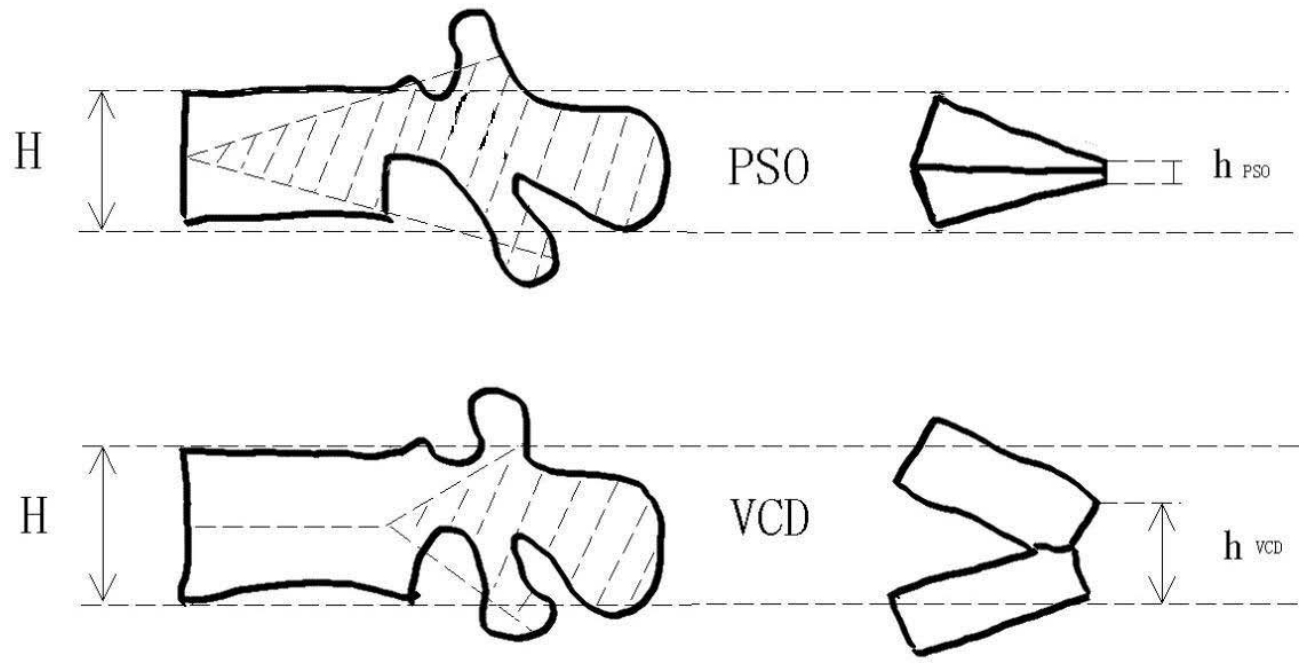

Figure 1: Comprative illustration of PSO and VCD procedures. The osteotomized angle of PSO is less than that of VCD. The middle column after VCD procedure is much greater than after PSO

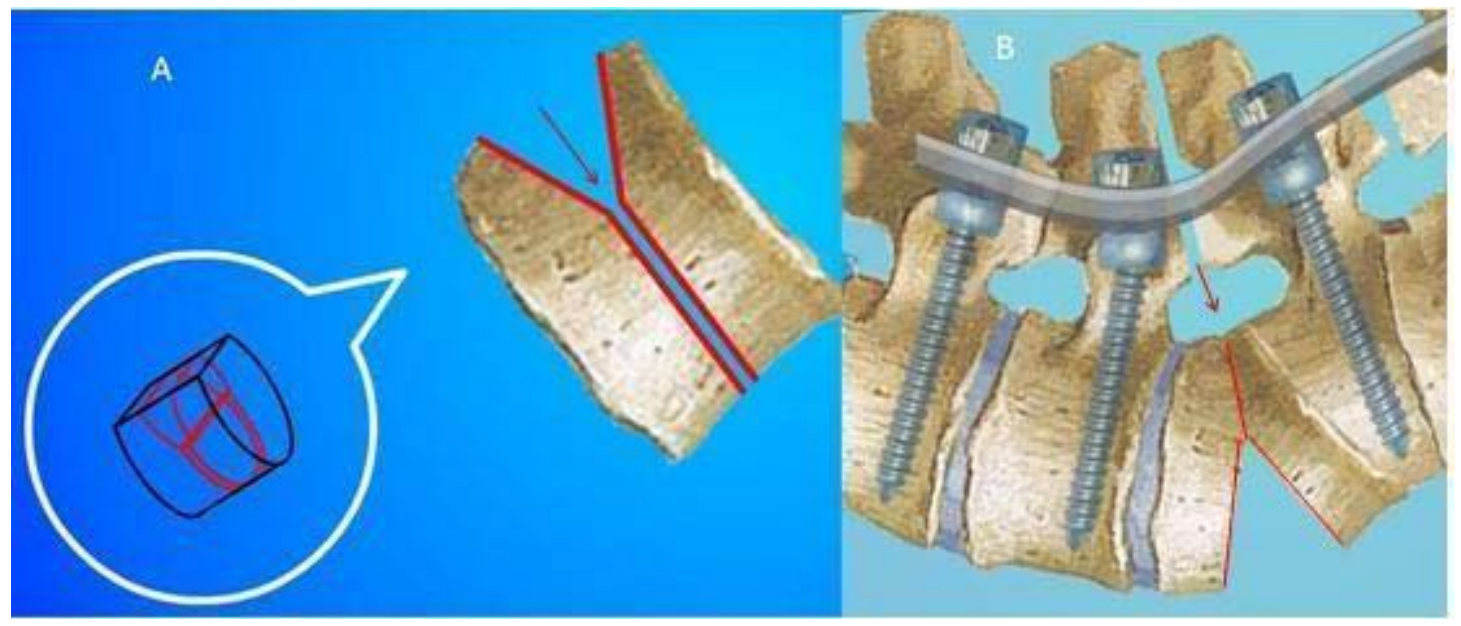

Figure 2: VCD Y-shaped osteotomy procedure A: The smaller wedge and the line-section compose Y-shaped osteotomy; it is a $360^{\circ}$ circumferential decancellation. B: The two surfaces of the wedge are closed and osteoclasis of the concave cortex occurred during correction 


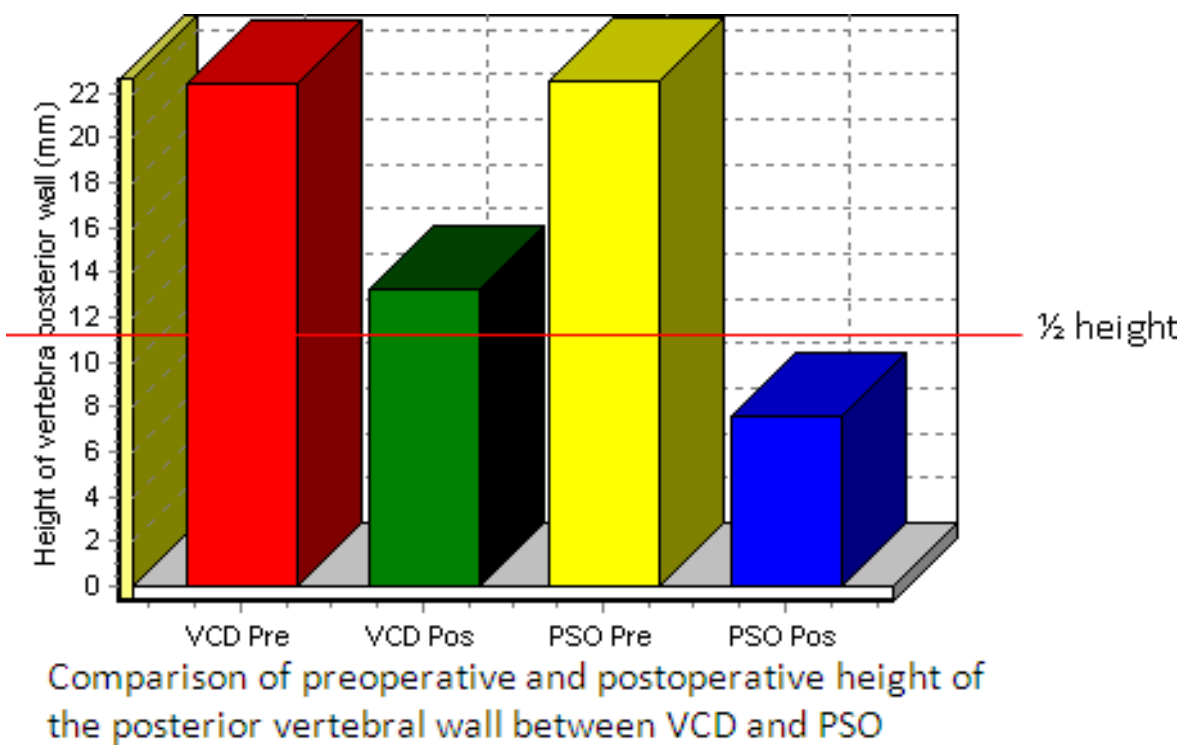

Figure 3: The postoperative height of VCD is $1 / 2$ the preoperative height, the height of PSO i $s \leq 1 / 3$ of the preoperative height. Height of the posterior wall of the resected vertebra after correction in VCD was greater than after PSO $(\mathrm{P}=0.0010)$
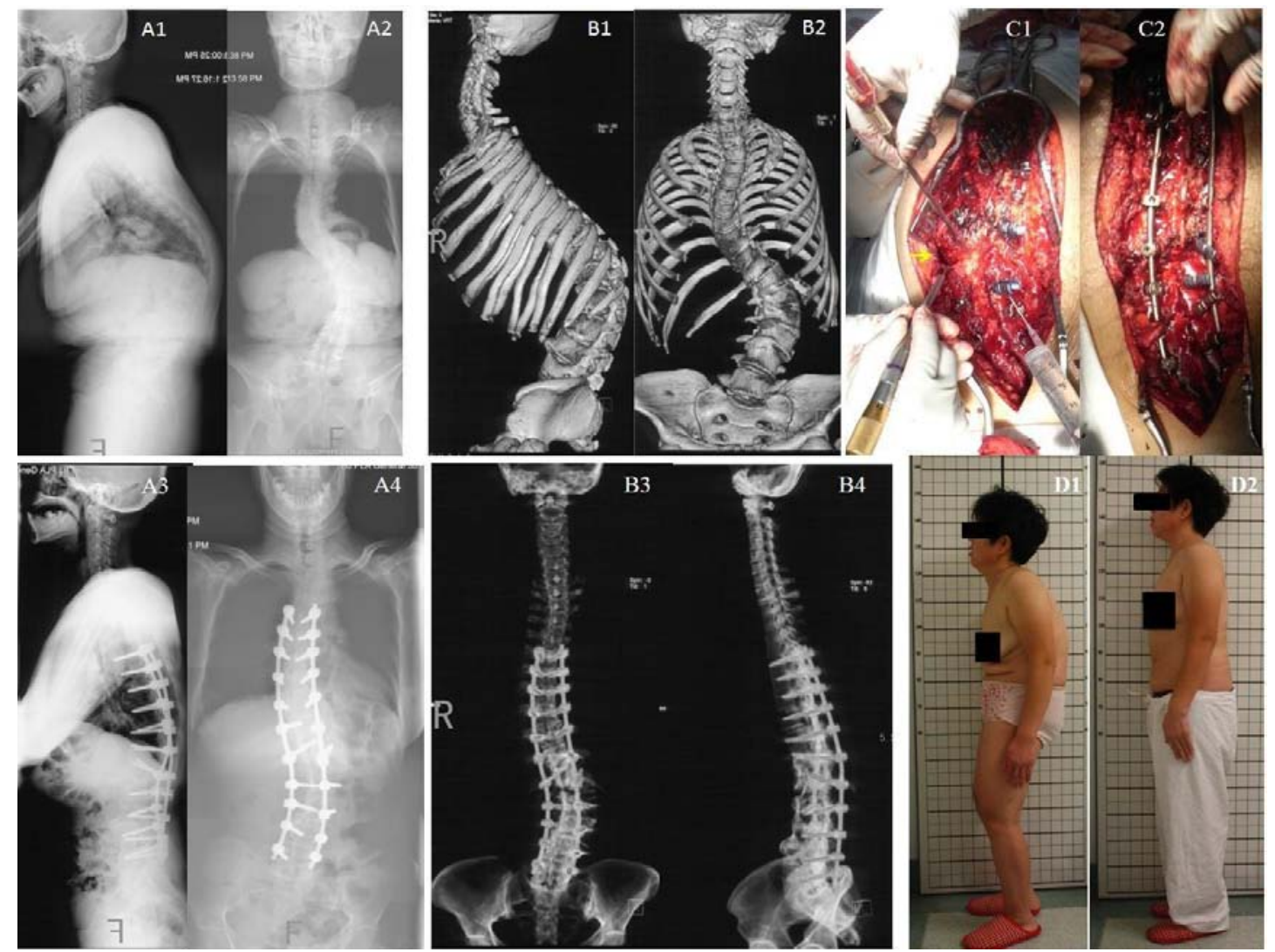

Figure 4: (A1-2) Standing lateral and anteroposterior radiographs of a 49-year-old female with a deformity of AS. (A34) The patient underwent L1 VCD osteotomy. (B1-2) anteroposterior and lateral CT s show the thoracolumbar deformities both in the sagittal and coronal planes. (B3-4) Sagittal and coronal balances were both restored. (C1-2) The approach of this VCD osteotomy was not a transpedicular resection but a transvertebral procedure, which has the advantage of obtaining a multidimensional correction (yellow arrow indicates the transvertebral site). (D1) Preoperative lateral view. (D2) Postoperative lateral view shows the correction of the deformity with VCD osteotomy at L1 

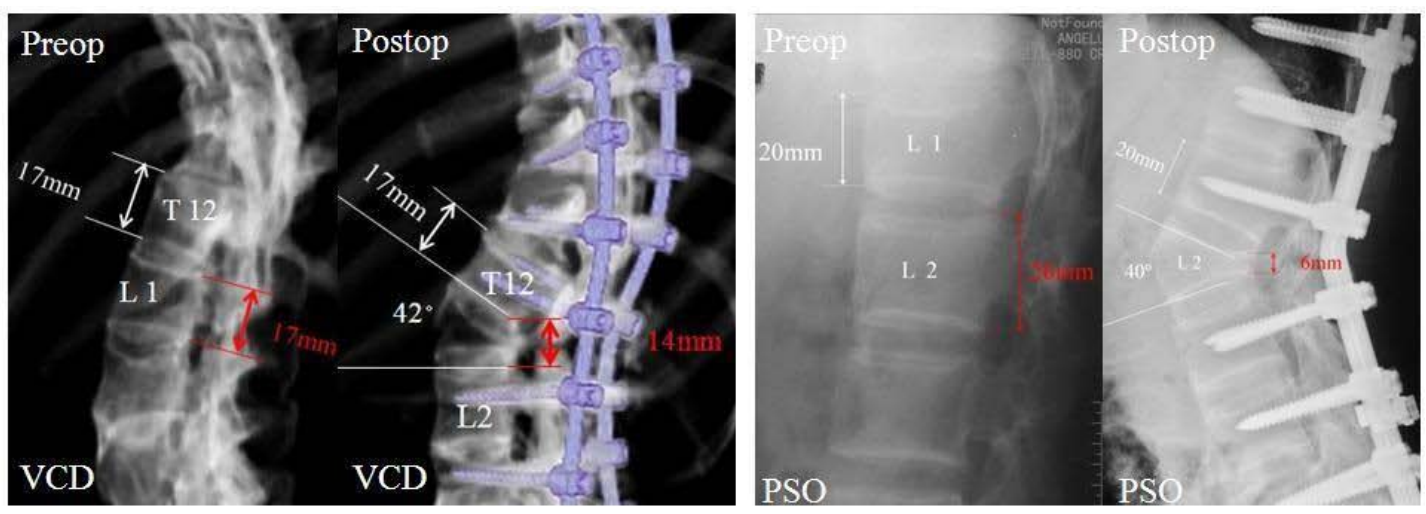

Figure 5: These two patients underwent VCD and PSO. The preoperative and postoperative heights of the posterior wall of the resected vertebras were measured (pre/postoperatively: VCD 17/14 mm, PSO 20/6 mm)

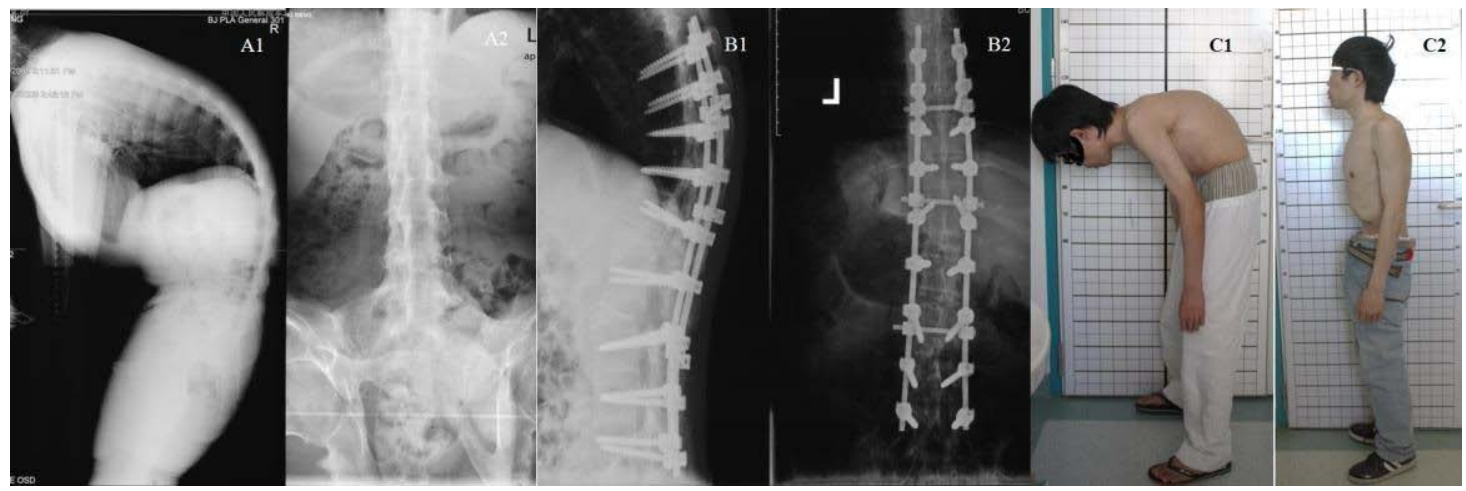

Figure 6: A 23-year-old male with AS kyphosis deformity secondary to AS underwent a 2-level PSO correction. (A1-2) Preoperative standing lateral and anteroposterior radiographs, the patient then underwent L1 and L3 PSO. (B1-2) Postoperative lateral and anteroposterior radiograph
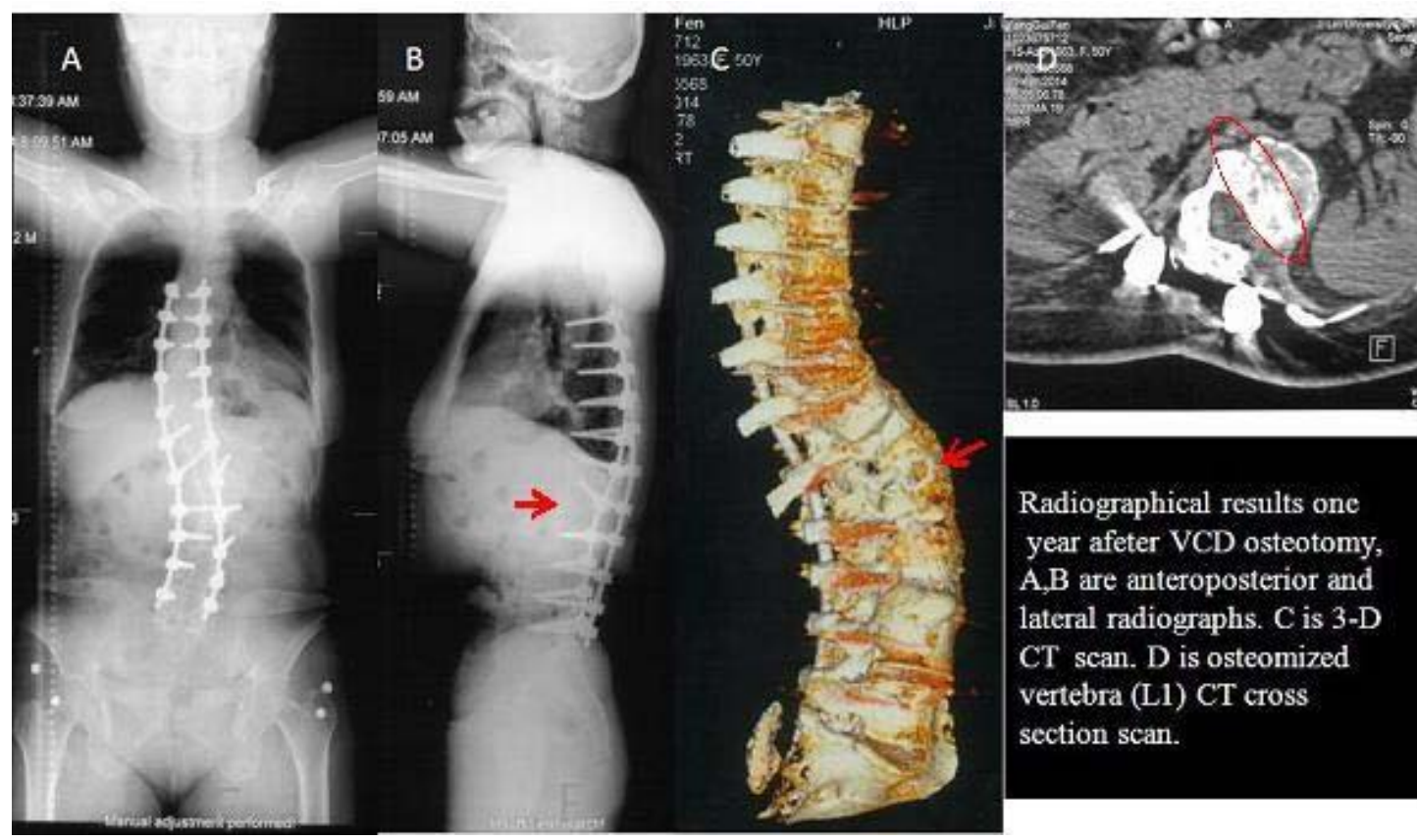

Figure 7: One year follow up radiographs and CTs of the 49 female patient underwent VCD osteotomy in Figure 4. Osteotomised segment was marked by red arrow in B and C, and bony healing area was observed in $\mathrm{C}$ which marked by red ring 